\title{
VALORACIÓN NUTRICIONAL DE LAS PERSONAS MAYORES DE 60 AÑOS DE LA CIUDAD DE PASTO, COLOMBIA ${ }^{1}$
}

\section{NUTRITIONAL ASSESSMENT OF PEOPLE OVER THE AGE OF 60 IN PASTO CITY, COLOMBIA}

\author{
Míldred Rosero Otero* \\ Giovana Marcela Rosas Estrada ${ }^{* *}$
}

\begin{abstract}
RESUMEN
La malnutrición es un problema que está más comúnmente reconocida entre las poblaciones que envejecen, aumentando su morbilidad y mortalidad. Objetivo: Determinar el estado nutricional de los adultos mayores de 60 años de Pasto, Colombia y su relación con variables sociodemográficas. Material y método: Estudio observacional descriptivo. La población estuvo conformada por los adultos mayores de la ciudad de Pasto, 45.953 adultos. La muestra fue de 426. El tipo de muestreo fue no probabilístico por conveniencia por cuotas estratificado por edad y sexo. Se utilizó una encuesta para la obtención de datos sociodemográficos y el Test Mini Nutritional Assessment (MNA) para la valoración nutricional. Resultados: Los participantes en un $61,7 \%$ fueron mujeres, el promedio de edad fue de 72 años; en la valoración nutricional el mayor porcentaje $(60,1 \%)$ está en riesgo de malnutrición, el 31,8\%, tiene un estado nutricional normal y el 8,1\% tiene malnutrición. Hubo diferencias significativas en MNA según nivel educativo y estrato socioeconómico. Conclusión: Los adultos mayores residentes en la comunidad de la ciudad de Pasto presentan riesgo de malnutrición de acuerdo al MNA. Se encontró asociación entre la malnutrición, la baja escolaridad y bajos estratos socioeconómicos. Es vital intervenir a esta población de forma interdisciplinaria ya que esta condición predispone otras situaciones que alteran la salud del adulto mayor como deterioro cognitivo y fragilidad. Es fundamental considerar que el estado nutricional es uno de los diagnósticos de enfermería más utilizados en la práctica y se hace necesario valorarlo en las personas mayores de 60 años.
\end{abstract}

Palabras clave: Nutrición del anciano, evaluación nutricional, estado nutricional, anciano, enfermería geriátrica.

\begin{abstract}
Malnutrition is a problem that is most commonly observed among aging populations, increasing their morbidity and mortality. Objective: To determine the nutritional status of adults over 60 years of age in Pasto, Colombia and its relationship with sociodemographic variables. Material and method: Observational, descriptive study. The population was made up of older adults from the city of Pasto, 45,953 adults. The sample was 426 . The type of sampling was a non-probabilistic, convenience sample with quotas stratified by age and sex. A survey was used to obtain sociodemographic data and the Mini Nutritional Assessment Test used for nutritional assessment. Results: $61.7 \%$ of the participants were women, the average age was 72 years. In the nutritional

\footnotetext{
${ }^{1}$ Financiamiento: Universidad Mariana, Acuerdo 004 de 28 enero de 2015 del Consejo Académico.

* Enfermera Magíster en Salud Ocupacional, Doctora en Enfermería de la Universidad Andrés Bello de Santiago de Chile, Docente Titular Universidad Mariana, Pasto, Nariño, Colombia. Email: mileroot2005@yahoo.es.

${ }^{* *}$ Fisioterapeuta Magíster en Gerontología, Envejecimiento y Vejez, Magíster en Intervención Integral en el Deportista, Doctoranda en Proyectos, UNINI México, Investigadora Grupo GIISE, Coordinadora de Investigación, Programa de Medicina, Universidad Cooperativa de Colombia, sede Pasto, Nariño, Colombia. Email: giovanarosas@gmail.com
} 
assessment the highest percentage (60.1\%), is at risk of malnutrition, $31.8 \%$, has a normal nutritional status and $8.1 \%$ suffers from malnutrition. There were significant differences in MNA according to educational level and socioeconomic stratum. Conclusion: Older adults living in the community of the city of Pasto, are at risk of malnutrition according to MNA. A relation was found between malnutrition and low educational an socioeconomic level. It is vital to intervene in this population in an interdisciplinary manner since this condition predisposes other situations that alter the health of the elderly such as cognitive deterioration and frailty. It is fundamental to consider that the nutritional status is one of the most used nursing diagnoses in practice and it is necessary to assess it in people over 60 years of age.

Key words: Elderly nutrition, nutritional assessment, nutritional status, elderly, geriatric nursing.

Fecha recepción: 23/11/16 Fecha aceptación: 28/12/17

\section{INTRODUCCIÓN}

El mundo actualmente presenta un crecimiento acelerado de la población adulta mayor; hasta el año 2000 existían 606 millones de adultos mayores en todo el mundo y para el 2050 se espera que sean aproximadamente 1,2 billones (1). Según el Departamento $\mathrm{Na}$ cional de Estadística (DANE), Colombia en el año 2010 contaba con 4.473 .447 adultos mayores de 60 años de edad, para el 2020 se espera una población, con el mismo rango de edad, mayor a 6.435 .899 y se calcula que para el 2050 habrá 15 millones de personas en esa edad (2). En el contexto regional, el Departamento de Nariño (Colombia) no es ajeno al cambio demográfico, la población total, según las proyecciones del DANE Censo 2005 para el año 2013, es de 1.701.782 habitantes que representa el 3,61\% de la población nacional; en este orden, la concentración departamental de personas de 60 años y más es del 10,30\% (175.352 personas mayores) y para personas en proceso de envejecimiento (45 a 59 años) es del $13.71 \%$ (233.350 personas), distribuidas en las 13 subregiones del Departamento (3).

Este cambio demográfico hace que la atención en salud hacia esta población sea cada vez más significativa y se haga de manera integral. Uno de los aspectos que hay que valorar en este grupo etáreo es la nutrición ya que el envejecimiento viene acompañado de cambios fisiológicos que pueden afectar el estado nutricional. Las deficiencias sensoriales, tales como un menor sentido del gusto o del olfato, o ambos, en muchos casos disminuyen el apetito. La mala salud bucodental o los problemas dentales pueden producir dificultad para masticar, inflamación de las encías y una dieta monótona de baja calidad, factores que aumentan el riesgo de desnutrición (4). Asimismo, se ve afectada la secreción de ácido gástrico, que reduce la absorción de hierro y vitamina B12. Otros cambios como la pérdida progresiva de visión y audición, así como la artrosis, en muchos casos limitan la movilidad y afectan la capacidad de las personas mayores para ir a comprar alimentos y preparar comidas (5).

El envejecimiento también puede venir acompañado de cambios psicosociales y ambientales, como el aislamiento, la soledad, la depresión y la falta de ingresos, lo que influye de manera importante en la dieta (6). Lo anterior aumenta el riesgo de desnutrición en la vejez, la que se manifiesta en reducción de la masa muscular y ósea, mayor riesgo de fragilidad (7), deterioro de la función cognitiva, deterioro de la capacidad para cuidar de sí mismo y mayor dependencia (8).

Por otra parte hay una relación muy estrecha entre nutrición y salud. La malnutrición es uno de los problemas clínicos más frecuentes entre la población de la tercera edad, el déficit de peso y la desnutrición es una complicación muy común en edades avanza- 
das y muchas veces la evaluación de los mayores plantea desafíos a los profesionales de la salud porque los indicios de un problema nutricional también pueden ser el resultado de causas no nutricionales $(9,10)$. Un estado nutricional inadecuado en esta etapa provoca además pérdida en los mecanismos de vitalidad; aumentando la vulnerabilidad ante cualquier tipo de agresión, incluyendo las enfermedades mentales como la depresión y/o aparición de síntomas depresivos (11).

En adición a lo anterior, cabe mencionar que el estado nutricional es uno de los diagnósticos de enfermería más utilizados en la práctica clínica para atender las respuestas humanas que se presentan en las personas con alteraciones en el mismo. Por ello es necesario explorar el estado nutricional y la ingesta dietética en el adulto mayor (12), además, la nutrición es vista como el factor más influyente en la salud de los adultos mayores (13) y debe ser un aspecto vital a tener en cuenta en la intervención por parte del personal de enfermería, promoviendo en esta población la realización de actividad física regular, la disminución del consumo de alcohol o tabaco y la adopción de una dieta saludable adaptada a las necesidades propias del envejecimiento (14).

El personal de enfermería es un actor fundamental dentro del proceso de evaluación y asesoramiento dietético; su cercanía con el paciente permite fomentar la confianza e implementar los cambios sugeridos para promover el autocuidado $(15,16)$, de ahí que el asesoramiento nutricional debe enfocarse a todas las áreas de intervención tanto a nivel clínico como comunitario ya que es el profesional de enfermería el encargado de hacer la valoración nutricional inicial identificando posibles alteraciones que requieran una intervención más a fondo en el aspecto nutricional, utilizando instrumentos para su valoración como el Test Mini Nutritional Assessment (MNA) que le permitirá conocer de manera ágil el estado nutricional en que se encuentra el adulto mayor (14).
Por lo anterior, la presente investigación buscó determinar el estado nutricional de los adultos mayores de la ciudad de Pasto y su relación con variables sociodemográficas, con el uso del Test Mini Nutritional Assessment (MNA) que es el método más utilizado en los últimos años en la valoración nutricional de la población anciana (17).

\section{MATERIAL Y MÉTODO}

El presente estudio fue realizado en la ciudad de Pasto, Nariño, Colombia, con adultos mayores no institucionalizados. El paradigma utilizado fue el cuantitativo, el diseño fue de tipo observacional descriptivo. La población estuvo conformada por los adultos mayores de la ciudad de Pasto, 45.953 adultos según datos del Censo DANE 2005 y su estimación para el año 2013. La muestra fue de 382 personas, ajustándose este valor con un factor de no respuesta del $11 \%$, obteniéndose finalmente una muestra de 426 adultos mayores. El tipo de muestreo fue no probabilístico por conveniencia por cuotas estratificado por edad y sexo. Los sujetos se seleccionaron de cada una de las 12 comunas de la ciudad y que cumplían los siguientes criterios de inclusión: ser mayores de 60 años y que no presenten alguna condición de inestabilidad clínica o hemodinámica que impidiera su participación.

Los instrumentos utilizados fueron la encuesta para la obtención de datos sociodemográficos y el Test Mini Nutritional Assessment para la valoración nutricional, los cuales fueron previamente piloteados.

Existen varios instrumentos para el despistaje de riesgo nutricional y de desnutrición, siendo el "Mini Nutritional Assessment" (MNA) el más utilizado y validado en diversas lenguas, que considera parámetros antropométricos, preguntas de autoevaluación y una encuesta nutricional (18). El MNA es un instrumento validado internacionalmente 
que permite una valoración del estado nutricional del anciano de forma sencilla, práctica y breve y se utiliza en ancianos ambulatorios, o con cuidados en casa y hospitalizados (1922). Esta prueba presenta unos excelentes índices de validez diagnóstica con elevada sensibilidad (96\%) y especificidad (98\%) (23), y detecta el riesgo de malnutrición antes de que aparezcan las alteraciones clínicas (valor predictivo para la malnutrición del 97\%). Así mismo suministra una evaluación global del estado de salud de los mayores de 64 años, pues además incluye la valoración cognitiva y el estado funcional y dietético de la persona. El MNA consta de 18 ítems divididos en 4 categorías: parámetros antropométricos, estado general del paciente, encuesta dietética y valoración subjetiva. De este modo, la prueba clasifica a las personas mayores, sobre un máximo de 30 puntos, en tres grupos: estado nutricional satisfactorio (S) ( $\geq 24$ puntos), riesgo de malnutrición (RM) (17-23,5 puntos) y malnutrición (M) (<17 puntos) (23).

Los adultos mayores no institucionalizados participantes de esta investigación fueron valorados con el MNA por estudiantes de enfermería y medicina previamente entrenados y capacitados en la aplicación de este instrumento, además fueron supervisados por los investigadores integrantes del presente proyecto. Los adultos pertenecientes a las 12 comunas de la ciudad de Pasto eran citados a diferentes lugares que con anterioridad se elegían entre los que se encontraban: colegios, salones comunales, canchas deportivas, salones parroquiales y auditorios, entre otros. En total se realizaron once jornadas de campo y es importante destacar que antes de aplicar el MNA, los participantes firmaban el consentimiento informado como una de las consideraciones éticas que se tuvo en cuenta en la investigación. La investigación fue aprobada por el Comité de Ética bajo Acuerdo 004 del 28 de enero de 2015.

La información fue almacenada en Microsoft Excel y se utilizó el software estadístico SPSS versión 19. Para el análisis se utilizó es- tadística descriptiva y Prueba t. Para establecer la diferencia por sexo, edad, nivel educativo y estrato socioeconómico en la variable nutrición, se utilizó tablas de contingencia, con la respectiva prueba de Chi cuadrado.

\section{RESULTADOS}

En la Tabla 1 se puede observar que en un mayor porcentaje $(61,7 \%)$, los adultos mayores son mujeres, las edades predominantes se encuentran en los rangos de 65 a 69 años $(24,4 \%)$ y 70 a 74 años $(24 \%)$, con una media de 72 años (DE 7), una edad mínima de 60 años y máxima de 94 años.

Los valores antropométricos de la muestra según sexo se muestran en la Tabla 2. Los parámetros de peso y talla ofrecieron los siguientes resultados: las mujeres presentaron un peso medio de 60,0 (DE 11,2) kg y una talla de 147,8 (DE 10,0) cm, mientras que dichos valores en los hombres fue 64 (DE 9,8) kg y 159,3 (DE 9,7) cm, respectivamente, con diferencias altamente significativas $(\mathrm{p}<0,01)$. El índice de masa corporal (IMC) para la muestra fue de 27,2 (DE 9,6) kg/m². En las mujeres el valor fue 27,9 (DE 9,5) $\mathrm{kg} / \mathrm{m}^{2}$ frente a $26,1($ DE 9,6$) \mathrm{kg} / \mathrm{m}^{2}$ para los hombres (p0,05). Respecto al valor del IMC, los resultados han mostrado que el IMC de las personas que son estudiadas es significativamente mayor en las mujeres frente a los hombres. Los resultados de la comparación entre varones y mujeres en la Circunferencia Branquial (CB) y Circunferencia de la Pantorrilla $(\mathrm{CP})$ no resultaron significativos.

La pérdida de peso en los últimos 3 meses no presentó diferencias significativas por sexo según la prueba Chi cuadrado (Tabla 3).

Los resultados globales del MNA evidenciaron que el 60,1\% (193) de los adultos mayores evaluados están en riesgo de malnutrición, el 31,8\% (102) tienen una nutrición normal y el 8,1\% (26) están clasificados con malnutrición. 
Tabla 1. Distribución de frecuencias de las variables sexo y edad en la muestra de 426 personas mayores de 60 años en la ciudad de Pasto.

\begin{tabular}{llrc}
\hline \multicolumn{2}{l}{ Variables } & $\mathrm{n}$ & $\%$ \\
\hline Sexo & Mujer & 263 & 61.7 \\
& Hombre & 163 & 38.3 \\
Edad & $60-64$ & 68 & 16.8 \\
& $65-69$ & 99 & 24.4 \\
& $70-74$ & 97 & 24.0 \\
& $75-79$ & 63 & 15.6 \\
& 80 o más & 78 & 19.3 \\
\hline Total & & 426 & 100 \\
\hline
\end{tabular}

Tabla 2. Distribución de promedios, desviación estándar y frecuencias de variables de la Escala Mini Nutritional Assessment (MNA), por sexo de la muestra de 426 personas mayores de 60 años en la ciudad de Pasto.

\begin{tabular}{lcccrc}
\hline \multirow{2}{*}{ Variables } & \multicolumn{2}{c}{ Media (DE) } & Media general & & \\
\cline { 2 - 3 } & Mujer & Hombre & $(\mathrm{DE})$ & $\mathrm{t}$ & p valor \\
\hline Peso & $60,0(11,2)$ & $64,9(9,8)$ & $61,9(11,0)$ & 4,60 & 0 \\
Talla & $147,8(10,0)$ & $159,3(9,7)$ & $152,2(11,3)$ & 11,67 & 0 \\
Índice de Masa Corporal & $27,9(9,5)$ & $26,1(9,6)$ & $27,2(9,6)$ & 1,89 & 0,05 \\
Circunferencia braquial & $28,5(3,7)$ & $28,2(3,1)$ & $28,4(3,4)$ & 0,86 & 0,311 \\
Circunferencia de la pantorrilla & $34,3(5,3)$ & $34,8(5,0)$ & $34,5(5,5)$ & 0,97 & 0,371 \\
\hline
\end{tabular}

Tabla 3. Distribución de frecuencias de pérdida de peso por sexo en los últimos 3 meses en la muestra de 426 personas mayores de 60 años en la ciudad de Pasto.

\begin{tabular}{ccccccccc}
\hline & \multicolumn{3}{c}{ Pérdida de Peso (<3 meses): } & & \\
\cline { 2 - 5 } Sexo & No Sabe & Sin pérdida & $\begin{array}{c}\text { Entre } \\
1 \text { y } 3 \mathrm{~kg}\end{array}$ & $\begin{array}{c}\text { Mayor a } \\
3 \mathrm{~kg}\end{array}$ & Total & Chi $^{2}$ & p valor \\
\hline Mujer & $52(19,8)$ & $120(45,6)$ & $63(24,0)$ & $28(10,6)$ & $263(100,0)$ & 0,86 & 0,804 \\
Hombre & $32(19,6)$ & $70(42,9)$ & $39(23,9)$ & $22(13,5)$ & $163(100,0)$ & & \\
\hline
\end{tabular}

En la Tabla 4 se presentan los resultados obtenidos en las variables sobre valoración subjetiva sobre problemas de nutrición y estado de salud distribuidos por sexo. Es destacable que las mujeres tienen una mayor percepción de malnutrición severa o moderada $(57 \%)$ frente a los hombres (40\%), con diferencias estadísticas altamente significati- vas $(\mathrm{p}<0,01)$, según la prueba Chi cuadrado. En la percepción sobre el estado de salud las diferencias por sexo no fueron significativas.

Se determinó que el Nivel Educativo y Estrato Social son factores asociados al estado Nutricional ( $p<0,05$, prueba Chi cuadrado), presentando mayor frecuencia de malnutrición o riesgo de malnutrición los ancianos 
con niveles más bajos de educación y estrato socioeconómico, como se observa en la Tabla 5. Igualmente se observó más altas frecuencias de malnutrición en las mujeres que en los hombres, aunque las diferencias no fueron tan significativas $(\mathrm{p}=0,072$, prueba Chi cuadrado). Por grupos de edad no se detectaron diferencias en el estado nutricional.
Por otra parte, de la Tabla 6 se infiere que los ancianos con percepciones negativas de nutrición y de salud presentan mayores frecuencias de malnutrición y riesgo de malnutrición que aquellos ancianos con percepciones más positivas, siendo estas diferencias altamente significativas $(\mathrm{p}<0,01$, prueba Chi cuadrado).

Tabla 4. Valoración subjetiva sobre nutrición y estado de salud, según sexo, en la muestra de 426 personas mayores de 60 años en la ciudad de Pasto.

\begin{tabular}{|c|c|c|c|c|c|}
\hline & & \multicolumn{2}{|c|}{ Frecuencia n (\%) } & \multirow{2}{*}{$\mathrm{Chi}^{2}$} & \multirow{2}{*}{$\mathrm{p}$ valor } \\
\hline & & Mujer & Hombre & & \\
\hline \multirow{4}{*}{$\begin{array}{l}\text { Problemas de } \\
\text { Nutrición }\end{array}$} & Malnutrición severa & $9(3,4)$ & $5(3,1)$ & \multirow{4}{*}{12,77} & \multirow{4}{*}{0,003} \\
\hline & Malnutrición moderada & $140(53,2)$ & $59(36,9)$ & & \\
\hline & Sin problemas & $112(42,6)$ & $96(60,0)$ & & \\
\hline & No lo sabe & $2(0,8)$ & $3(1,8)$ & & \\
\hline \multirow{4}{*}{$\begin{array}{l}\text { Estado de } \\
\text { Salud }\end{array}$} & Peor & $45(17,1)$ & $19(11,8)$ & \multirow{4}{*}{6,96} & \multirow{4}{*}{0,073} \\
\hline & Igual & $69(26,2)$ & $32(19,9)$ & & \\
\hline & Mejor & $118(44,9)$ & $93(57)$ & & \\
\hline & No lo sabe & $30(11,8)$ & $17(10,6)$ & & \\
\hline
\end{tabular}

Tabla 5. Distribución de frecuencias del Estado Nutricional según puntaje del MNA por sexo, grupos de edad, nivel educativo y estrato socioeconómico en la muestra de 426 ancianos de la ciudad de Pasto.

\begin{tabular}{|c|c|c|c|c|c|c|c|}
\hline \multirow{2}{*}{ Variables } & & \multicolumn{3}{|c|}{ Clasificación Mini Nutrición n (\%) } & \multirow[b]{2}{*}{ Total n (\%) } & \multirow[b]{2}{*}{$\mathrm{Chi}^{2}$} & \multirow[b]{2}{*}{$\mathrm{p}$ valor } \\
\hline & & Malnutrición & $\begin{array}{c}\text { Riesgo } \\
\text { Malnutrición } \\
\end{array}$ & Normal & & & \\
\hline \multirow[t]{2}{*}{ Sexo } & Mujer & $21(10,1)$ & $128(61,5)$ & $59(28,4)$ & $208(100,0)$ & \multirow{2}{*}{6,36} & \multirow{2}{*}{0,072} \\
\hline & Hombre & $5(4,4)$ & $65(57,5)$ & $43(38,1)$ & $113(100,0)$ & & \\
\hline \multirow[t]{5}{*}{ Grupos de edad } & $60-64$ & $2(3,6)$ & $34(60,7)$ & $20(35,7)$ & $56(100,0)$ & \multirow{5}{*}{7,92} & \multirow{5}{*}{0,442} \\
\hline & $65-69$ & $7(9,0)$ & $51(65,4)$ & $20(25,6)$ & $78(100,0)$ & & \\
\hline & $70-74$ & $5(7,0)$ & $43(60,6)$ & $23(32,4)$ & $71(100,0)$ & & \\
\hline & $75-79$ & $4(7,8)$ & $34(66,7)$ & $13(25,5)$ & $51(100,0)$ & & \\
\hline & $80 \mathrm{o}+$ & $6(11,8)$ & $24(47,1)$ & $21(41,2)$ & $51(100,0)$ & & \\
\hline \multirow[t]{4}{*}{ Nivel educativo } & Ninguno & $12(14,6)$ & $51(62,2)$ & $19(23,2)$ & $82(100,0)$ & \multirow{4}{*}{12,93} & \multirow{4}{*}{0,004} \\
\hline & Primaria & $12(6,5)$ & $108(58,1)$ & $66(35,5)$ & $186(100,0)$ & & \\
\hline & Secundaria & $2(4,9)$ & $29(70,7)$ & $10(24,4)$ & $41(100,0)$ & & \\
\hline & Universitaria & $0(0,0)$ & $5(45,5)$ & $6(54,5)$ & $11(100,0)$ & & \\
\hline \multirow[t]{3}{*}{ Estrato Socioeconómico } & Bajo & $25(10,0)$ & $156(62,4)$ & $69(27,6)$ & $250(100,0)$ & \multirow{3}{*}{15,53} & \multirow{3}{*}{0,044} \\
\hline & Medio & $1(1,7)$ & $32(55,2)$ & $25(43,1)$ & $58(100,0)$ & & \\
\hline & Alto & $0(0,0)$ & $1(16,7)$ & $5(83,3)$ & $6(100,0)$ & & \\
\hline
\end{tabular}


Tabla 6. Valoración subjetiva sobre nutrición y estado de salud en la muestra de 426 ancianos de la ciudad de Pasto.

\begin{tabular}{llcccccc}
\hline & Variables & \multicolumn{2}{c}{ Clasificación Mini Nutrición n (\%) } \\
& Malnutrición & $\begin{array}{c}\text { Riesgo de } \\
\text { Malnutrición }\end{array}$ & Normal & Totaln $(\%)$ & Chi $^{2}$ & p valor \\
\hline Problemas de & Malnutrición severa & $6(60,0)$ & $4(40,0)$ & $0(0,0)$ & $10(100,0)$ & \\
Nutrición & Malnutrición moderada & $20(12,4)$ & $119(73,9)$ & $22(13,7)$ & $161(100,0)$ & 89,8 & 0,000 \\
& Sin problemas & $0(0,0)$ & $70(46,7)$ & $80(53,3)$ & $150(100,0)$ & \\
Estado de & Peor & $18(32,7)$ & $33(60,0)$ & $4(7,3)$ & $55(100,0)$ & \\
Salud & igual & $2(2,6)$ & $60(76,9)$ & $16(20,5)$ & $78(100,0)$ & 240,91 & 0,000 \\
& Mejor & $2(1,3)$ & $71(47,0)$ & $78(51,7)$ & $151(100,0)$ & \\
& No lo sabe & $4(10,8)$ & $29(78,4)$ & $4(10,8)$ & $37(100,0)$ & \\
\hline
\end{tabular}

\section{DISCUSIÓN Y CONCLUSIÓN}

Diversos estudios experimentales y epidemiológicos han mostrado que el estado nutricional de la población en general, y de los ancianos en particular, es un indicador válido para predecir tanto la longevidad como la calidad de vida en esta etapa del ciclo vital (24-26). La Organización Mundial de la Salud (OMS) en el estudio de nutrición en el envejecimiento, señaló a la población anciana como un grupo nutricionalmente muy vulnerable, debido a los cambios anatómicos y fisiológicos asociados al proceso de envejecimiento (27). Se estima que aproximadamente el 35-40\% de los ancianos presenta algún tipo de alteración nutricional como: malnutrición calórico-proteica, déficit selectivo de vitaminas y/o micronutrientes, inadecuado aporte hídrico y obesidad. De igual manera la OMS en su Informe Mundial sobre el Envejecimiento y la Salud (5) afirma que los cambios propios del envejecimiento a nivel fisiológico, psicosocial y ambiental pueden tener efectos importantes en la dieta del adulto mayor, aumentando el riesgo de desnutrición en la vejez, lo que a menudo se manifiesta en reducción de la masa muscular y ósea y mayor riesgo de fragilidad. La desnu- trición también se ha asociado con deterioro de la función cognitiva, deterioro de la capacidad para cuidar de uno mismo y mayor riesgo de dependencia de cuidados. En este informe se menciona que la desnutrición en la vejez a menudo no se diagnostica, y son pocas las evaluaciones exhaustivas de la prevalencia mundial de las diferentes formas de desnutrición.

Por otra parte, la feminización del envejecimiento es un comportamiento que es similar en muchos lugares. Estudios demuestran que el sexo femenino prevalece en los adultos mayores $(28,29)$, esto sucede de la misma manera en los ancianos de Pasto que viven en comunidad o en su domicilio y que fueron objeto del presente estudio.

En la ciudad de la Habana (Cuba), en el estudio realizado con ancianos atendidos en 3 escenarios diferentes: comunidad, servicio de geriatría y hogar de ancianos, se valoró el estado nutricional y se estimó las tasas de desnutrición a través del test Mini Nutritional Assessment (MNA), los resultados arrojaron tasas dispares de trastornos nutricionales. La desnutrición fue virtualmente nula entre los adultos mayores que viven en la comunidad, pero llegó a ser casi universal entre aquellos internados en un Hogar de Ancianos (30). Los datos anteriores tienen distinto compor- 
tamiento con los encontrados en los ancianos de Pasto, ya que el 60,1\% de los adultos mayores están en riesgo de malnutrición, cifra significativa considerando la influencia de la nutrición en este grupo etario. Por otra parte, en el estudio cubano la desnutrición no parece ser un fenómeno epidemiológico importante entre los sujetos mayores de 60 años que viven en la comunidad (30). De hecho, el exceso de peso constituye el hallazgo nutricional más frecuente en tales poblaciones. El exceso de peso podría representar una de las facetas del Síndrome metabólico. En el estudio de González et al. (30), la frecuencia de trastornos nutricionales fue del 2,7\% entre los encuestados en la comunidad costera de Cojímar, pero se incrementó hasta ser del 91,6\% entre los atendidos en el Servicio de Geriatría, y del 95,3\% entre los sujetos institucionalizados en el Hogar de Ancianos, respectivamente. La ocurrencia de desnutrición puede ser baja entre los ancianos que viven en la comunidad, pero puede afectar a casi la totalidad de aquellos que acuden a las instituciones públicas de salud para ser atendidos (30).

En México, Shamah et al. (31) describieron el estado de salud y nutrición de los adultos mayores analizando los resultados de la Encuesta Nacional de Salud y Nutrición (ENSANUT 2006), encontrándose que los adultos mayores de 60 años de edad en México se caracterizan por estaturas muy bajas y una muy preocupante prevalencia de sobrepeso y obesidad. Una prevalencia de obesidad como la observada de casi $28 \%$ es sumamente preocupante, ya que es mayor a la observada en los Estados Unidos, donde alcanza 22,9\% en la población de 60 a 69 años y 15,5\% en mayores de 70; en realidad, también es mayor a la de algunos países de Latinoamérica y El Caribe, donde la prevalencia alcanza sólo 9,6\% (20) con excepción de las mujeres de Montevideo que tuvieron una prevalencia mayor $(40 \%)$. En un estudio realizado en la provincia española de Ourense por De la
Montaña et al. (32), se evaluó el riesgo nutricional mediante el MNA en una población anciana no institucionalizada mayores de 65 años, observándose que el 70\% de la población no presenta un estado nutricional adecuado (el 57,5\% está en situación de riesgo de desnutrición y el 12,5\% desnutridos); esta situación empeora entre las personas de 65 a 70 años y los mayores de 81 años y entre la población femenina. Los resultados del estudio de De la Montaña et al. se asemejan a los encontrados en la ciudad de Pasto, en el que predomina el sexo femenino en riesgo de malnutrición con un $61,5 \%$, con un rango de edad entre 65 a 69 años con el 65,4\%.

Otros datos similares al presente estudio son los encontrados en la investigación del estado nutricional de los ancianos de Cantabria, la recolección de datos fue realizada por personal de enfermería de colegios de enfermeras de Cantabria, este personal valoró el estado nutricional de personas mayores no hospitalizadas que eran atendidas por personal de atención primaria (en consulta a domicilio) o que estaban alojadas en una residencia; entre los datos más relevantes se tiene que la puntuación nutricional (PN) obtenida de la suma de los ítems del MNA fue 23,4 DE 4,1 para las mujeres y 24,4 DE 4 en los varones $(\mathrm{p}<0,001)$. Destaca el hecho de que el 22,3\% de las personas estudiadas en residencias está malnutrida o en riesgo de malnutrición, frente al 14,2\% de las que acuden a consulta, y sólo el 3,3\% de las estudiadas en su domicilio (28).

De igual forma, en el estudio realizado por Casas et al. (33) en Perú, en el cual describieron el perfil clínico del adulto mayor atendido ambulatoriamente en un hospital general y que arrojó datos similares a los de la ciudad de Pasto, se encontró que el 69,3\% de los adultos evaluados fueron mujeres; la edad promedio fue de 76,3 DE 8,9 años (rango: 60 a 100); $190(65,5 \%)$ tenían entre 60 a 79 años y $100(34,5 \%)$ fueron mayores de 80 años. Respecto al estado nutricional, 
el 31,4\% tenían riesgo de malnutrición y el $23,4 \%$ obesidad, con IMC no categorizado en intervalos pero con un valor $>$ a 30 para obesidad, el promedio fue de $23,4 \%$. Por otra parte la investigación que se llevó a cabo en la comunidad de Masma Chicche, Junín, que buscó determinar el estado nutricional y los factores asociados a malnutrición en el adulto mayor (21), presentó resultados muy semejantes a los de la presente investigación, con la diferencia que en Masma la mayoría de los adultos mayores fueron varones; la valoración nutricional indicó un porcentaje mayor hacia el riesgo de malnutrición, seguido de ancianos con malnutrición y un bajo porcentaje con nutrición normal.

Respecto al nivel educativo y el estrato social, en el presente estudio se encontró que estos factores están asociados al estado nutricional con diferencias significativas y asociación entre la malnutrición, la baja escolaridad y bajos estratos socioeconómicos, resultados similares se encontraron en un estudio realizado en Córdoba, Argentina, en el cual compararon el estado nutricional y el estado de salud en dos grupos de adultos mayores de diferentes condiciones de vida, observándose que éstas influyen en el estado nutricional y la salud de los adultos mayores; entre estas el nivel educativo y el estrato social (34). Otros estudios de igual manera evidencian alteraciones nutricionales asociadas a estas variables $(35,36)$, lo cual puede deberse a un inadecuado consumo de alimentos de buena calidad nutricional por su alto costo (37). De igual forma, en la ciudad de Chillán, Chile, se obtuvieron hallazgos en los cuales el estado nutricional normal fue predominante, asociándose a ser profesional y tener estudios superiores e ingresos altos (38). Pertenecer a un nivel socioeconómico bajo influye negativamente en el estilo de vida desde los hábitos alimentarios y el estado nutricional inadecuado (39). Es frecuente observar cómo el bajo nivel educativo aumenta las tasas de malnutrición por déficit o exceso $(35,40,41)$, de esta forma las personas con mayores capacidades económicas y educativas estarían en mejores condiciones para mantener una alimentación más adecuada y saludable.

En Colombia, en el corregimiento de Güintar del Municipio de Anzá, Antioquia, se evaluó el estado nutricional de un grupo de adultos mayores (42); aunque en este estudio la mayoría de los participantes tuvo una evaluación nutricional normal, se encontró que el riesgo de malnutrición fue mayor en las mujeres que en los hombres, igual que en la ciudad de Pasto; aunque la malnutrición fue mayor en los hombres en el estudio antioqueño. En cuanto a la valoración subjetiva sobre el estado de salud, los adultos mayores de Pasto en un mayor porcentaje consideran que su salud está mejor, al igual que en el estudio del Municipio de Anzá, que en un mayor porcentaje encuentran, de la misma manera, su salud mejor.

En conclusión, los adultos mayores de la ciudad de Pasto y que residen en sus domicilios presentan riesgo de malnutrición, en ellos prevalece el sexo femenino y las edades predominantes oscilan entre los 65 y 74 años. En cuanto a las medidas antropométricas, este grupo no tiene alteraciones significativas que indiquen desnutrición, sin embargo por los resultados globales del MNA es importante estudiar a fondo con otros indicadores nutricionales el estado de nutrición de estos adultos mayores para intervenir de forma rápida a esta población y disminuir la probabilidad de que se avance a situaciones mayores de alteraciones nutricionales. Se encontró asociación entre la malnutrición, la baja escolaridad y bajos estratos socioeconómicos.

Es relevante considerar además que la intervención de este grupo poblacional debe hacerse de forma multidisciplinaria, donde enfermería tiene un papel preponderante ya que la alteración del estado nutricional, en especial en la población anciana, es uno de los diagnósticos enfermeros más importantes en el proceso de atención de enfermería. 


\section{REFERENCIAS}

1. Bayarre V, Pérez P, Menéndez J. Las transiciones demográficas y epidemiológicas y la calidad de vida objetiva en la tercera edad. GeroInfo. 2006; 1(3): 1-35.

2. Departamento Administrativo Nacional de Estadística Colombia. (DANE). Proyecciones anuales de población por sexo, según grupos quinquenales de edad 1985-2020 [Internet]. Bogotá: DANE; 2005 [citado 30 jun 2017]. Disponible en: http://www.dane.gov.co.

3. Gobernación de Nariño, Colombia. Política Pública de Envejecimiento y Vejez del Departamento de Nariño 2013-2023 [Internet]. San Juan de Pasto: Gobernación de Nariño; 2015. [citado 30 jun 2017]. Disponible en: http:// nariño.gov.co/2012-2015/index.php/ eventos/6267-politica-publica-departamental-de-envejecimiento-y-vejez-2013-2023-en-el-departamento-de-narino

4. Kshetrimayum N, Reddy CV, Siddhana S, Manjunath M, Rudraswamy S, Sulavai S. Oral health-related quality of life and nutritional status of institutionalized elderly population aged 60 years and above in Mysore City, India. Gerodontology. 2013; 30(2): 119-25.

5. Organización Mundial de la Salud. Informe Mundial sobre el Envejecimiento y la Salud [Internet]. Ginebra: Organización Mundial de la Salud; 2015 [citado 17 nov 2017]. 282 p. Disponible en: https://goo. gl/iAh8qY

6. Varela L. Principios de Geriatría y Gerontología. 2a edición. Lima: Centro Editorial de la Universidad Peruana Cayetano Heredia; 2011. p. 501- 512.

7. Tello T, Varela L. Fragilidad en el adulto mayor: detección, intervención en la comunidad y toma de decisiones en el manejo de enfermedades crónicas. Rev Peru Med Exp Salud Publica [Internet] 2016 [citado 28 nov 2017]; 33(2). Disponible en: http://www.redalyc.org/articulo. oa?id=36346797019

8. León-Caballero MP, Alcolea-Martínez E. Estado nutricional en personas mayores y su influencia sobre el deterioro cognitivo y la demencia. Psicogeriatría. 2016; 6(3): 99-109.

9. Newman AB, Yanez D, Harris T, Duxbury A, Enright PL, Fried LP. Weight Change in old age and its association with mortality. J Am Geriatr Soc. 2001; 49(10): 1309-1318.

10. Zambrano R. Valoración nutricional del adulto mayor. La Salud de los adultos mayores una visión compartida. 2a edición. Washington, D.C.: OPS; 2011. p. 89-108.

11. Ribera C, Milán V, Ruiz R. Conceptos esenciales del envejecimiento. Medicine. 2006; 9(62): 4003-4010.

12. Celestino M, Salazar B, Novelo H. Nutrición y desempeño cognitivo del adulto mayor. Aquichán. 2009; 8(2): 159-169.

13. Moreno-Fergusson ME, Alvarado-García A. Adaptación y cuidado en el ser humano una visión de Enfermería: Adaptación y cuidado del anciano sano. Colombia: Manual Moderno; 2007. p. 222-44.

14. Alvarado-García A, Lamprea-Reyes L, Murcia-Tabares K. La nutrición en el adulto mayor: una oportunidad para el cuidado de enfermería. Enfermería Universitaria. 2017; 14(3): 199-206.

15. Boaz M, Rychani L, Barami K, et al. Nurses and nutrition: A survey of knowledge and attitudes regarding nutrition assessment and care of hospitalized elderly patients. J Contin Educ Nurs. 2013; 44(8): 357-64.

16. Young K, Bunn F, Trivedi D, Dickinson A. Nutritional education for community dwelling older people: A systematic review of randomized controlled trials. Int J Nurs Stud. 2011; 48(6): 751-80.

17. Gutiérrez LM, Picardi P, Aguilar SG, Avila JA, Menéndez J, Pérez AB. Gerontología y Nutrición del Adulto Mayor. México, 
D.F.: McGraw-Hill; 2010.

18. Varela LF. Nutrición en el adulto mayor. Rev Med Hered. 2013; 24: 183-185.

19. Cereda E. Mini nutritional assessment. Current Opin Clin Nutr Metab Care. 2012; 15(1): 29-41.

20. Skipper A, Ferguson M, Thompson K, Castellanos VH, Porcari J. Nutrition screening tools: An analysis of the evidence. JPEN J Parenter Enteral Nutr. 2012; 36(3): 292-298.

21. Contreras AL, Mayo GVA, Romaní DA, Tejada GS, Yeh M, et al. Malnutrición del adulto mayor y factores asociados en el distrito de Masma Chicche, Junín, Perú. Rev Med Hered. 2013; 24(3): 186-191.

22. Osuna IA, Verdugo S, Leal G, Osuna I. Estado nutricional en adultos mayores mexicanos: estudio comparativo entre grupos con distinta asistencia social. Rev Esp Nutr Hum Diet. 2015; 19(1): 12-20.

23. Vellas B, Guigoz Y, Garry PJ, Nourhashemi F, Bennahum D, Lauque S, Albarede JL. The Mini Nutritional Assessment (MNA) and its use in grading the nutritional state of elderly patients. Nutrition. 1999; 15(2): 116-122.

24. Petersen PE, Kandelman D, Arpin S, Ogawa H. Global oral health of older people-call for public health action. Community Dent Health. 2010; 27(4 Suppl 2): 257-67.

25. Risonar MG, Rayco-Solon P, Ribaya-Mercado JD, Solon JA, Cabalda AB, Tengco LW, et al. Physical activity, energy requirements, and adequacy of dietary intakes of older persons in a rural Filipino community. Nutr J [Internet]. 2009 [citado 28 nov 2017]; 8(19). Disponible en: https://www.ncbi.nlm.nih.gov/pmc/articles/PMC2689250/

26. Shahar S, Ibrahim Z, Fatah AR, Rahman SA, Yusoff NA, Arshad F, et al. A multidimensional assessment of nutritional and health status of rural elderly Malays. Asia Pac J Clin Nutr. 2007; 16(2): 346-53.

27. World Health Organization. Keep fit for life. Meeting the nutricional needs of older persons. Geneva: WHO; 2002.

28. Jiménez M, Sola JM, Pérez C, Turienzo MJ, Larrañaga G, Mancebo MA, et al. Estudio del estado nutricional de los ancianos de Cantabria. Nutr Hosp. 2011; 26(2): 345-354.

29. Jürschik P, Torres J, Román S, Nuin C, Botigué T. Estado nutricional de la población mayor de Cataluña de diferentes niveles asistenciales. Arch Latinoam Nutr. 2009; 59(1): 38-46

30. González Hernández A, Cuyá Lantigua M, González Escudero H, Sánchez Gutiérrez R, Cortina Martínez R, Barreto Penié $\mathrm{J}$, et al. Estado nutricional de ancianos diferentes: comunidad, servicio de geriatría, hogar de ancianos. Arch Latinoam Nutr. 2007; 57(3): 266-272.

31. Shamah-Levy T, Cuevas-Nasu L, Mundo-Rosas V, Morales-Ruán C, Cervantes-Turrubiates L, Villalpando-Hernández S. Estado de Salud y Nutrición de los adultos mayores en México: resultados de una encuesta probabilística nacional. Salud pública Méx. 2008; 50(5): 383-389.

32. De la Montaña J, Areal C, Míguez M. Evaluación del riesgo nutricional mediante el MNA en una población anciana no institucionalizada. Arch Latinoam Nutr. 2009; 59(4): 390-395.

33. Casas P, Varela L, Tello T, Ortiz P, Chávez H. Perfil clínico del adulto mayor atendido ambulatoriamente en un hospital general. Rev Med Hered. 2012; 23(4): 229234.

34. Acosta L, Carrizo E, Peláez E, Roque V. Condiciones de vida, estado nutricional y estado de salud en adultos mayores, Córdoba, Argentina. Rev Bras Geriatric Gerontol [Internet]. 2015 [citado 23 dic 2017]; 18(1). Disponible en: http://www. scielo.br/pdf/rbgg/v18n1/1809-9823-rbgg-18-01-00107.pdf

35. Schlüssel MM, Silva AA, Pérez-Escamilla R, Kac G. Household food insecurity and excess weight/obesity among Brazilian 
women and children: a life course approach. Cad Saude Publica 2013; 29(2): 219-26.

36. Martin MA, Lippert AM. Feeding her children, but risking her health: the intersection of gender, household food insecurity and obesity. Soc Sci Med 2012; 74(11): 1754-64.

37. Martín-Fernández J, Caillavet F, Lhuissier A, Chauvin P. Food insecurity, a determinant of obesity?: an analysis from a population-based survey in the Paris metropolitan area, 2010. Obes Facts 2014; 7(2): 120-9.

38. Sepúlveda P, Barrón V, Rodríguez A. Estado nutricional de adultos mayores activos y su relación con algunos factores sociodemográficos. Rev Cubana Salud Pública [Internet]. 2015 [citado 23 dic 2017]; 43(3). Disponible en: http://scielo.sld.cu/ pdf/rcsp/v43n3/spu05317.pdf

39. Lima Costa MF, Barreto SM, Firmo J,
Uchoa E. Socioeconomic position and health in a population of brazilian elderly: Bambuí Health and Aging Study (BHAS). Pan Am J Public Health. 2003; 13(6): 387-93.

40. Shariff ZM, Sulaiman N, Jalil RA, Yen WC, Yaw YH, Taib MN, et al. Food insecurity and the metabolic syndrome among women from low income communities in Malaysia. Asia Pac J Clin Nutr. 2014; 23(1): 138-47.

41. Situación de la niñez y del adulto mayor Julio-Agosto-Septiembre 2013. Lima: Instituto Nacional de Estadística e Informática (INEI); 2013.

42. Deossa-Restrepo GC, Restrepo-Betancur LF, Velásquez-Vargas JE, Varela Álvarez D. Evaluación nutricional de adultos mayores con el Mini Nutritional Assessment: MNA. Rev Univ. Salud. 2016; 18(3): 494504. 